\title{
Proton pump inhibitors: When is it too much of a good thing?
}

\author{
John Tshon Yit Soong ${ }^{1,2} P h D$
}

The popularity of proton pump inhibitors (PPIs) among both prescribers and patients may be ascribed to their relative low cost, efficacy and safety profile. Introduced in the late 1980s, PPIs are benzimidazole derivative drugs that inhibit the hydrogen-potassium ATPase exchanger ("the proton pump") on the luminal surface of the gastric parietal cell membrane. ${ }^{1}$ The inhibition of the exchanger mechanism strongly decreases the secretion of protons needed for the secretion of hydrochloric acid. The PPIs are clinically indicated for peptic ulcer disease, gastroesophageal reflux, Zollinger-Ellison syndrome, non-steroidal anti-inflammatory drug (NSAID)associated gut ulcers, and as a part of Helicobacter pylori eradication. Highly effective, their superiority over histamine-2 receptor antagonists $(\mathrm{H} 2 \mathrm{RAs})^{2}$ is due in part to their ability to maintain gastric $\mathrm{pH}>4$ reliably for up to 21 hours a day, but also to the phenomenon of tachyphylaxis observed in the action of H2RAs. ${ }^{3}$

However, PPIs are not a panacea for all ills. While not by any means conclusive, observational studies have suggested that long-term PPI use may be associated with important adverse effects. ${ }^{4}$ Systemically, such use has been associated with increased risks of osteoporotic fractures; malabsorption of vitamin $\mathrm{B}_{12}$, calcium, magnesium and iron; Clostridium difficile infection; ${ }^{5}$ dementia; pneumonia; kidney disease; and stroke. The local observed effects of prolonged gastric acid suppression include hypergastrinemia, gastric atrophy and polyp formation, chronic $H$. Pylori infection, ${ }^{6}$ and a potentially increased risk of gastric cancer. ${ }^{7}$

In Singapore, several studies have explored the magnitude of inappropriate PPI prescribing. A singlecentre, point prevalence study found that $46.5 \%$ of hospitalised inpatients were prescribed a PPI, of which just over half (54.1\%) did not have a US Food and Drug Administration (FDA) approved clinical indication. ${ }^{8}$ Another Singapore study of hospitalised seniors ( $>65$ years) suggests that the rate of potentially inappropriate prescribing of PPI is even higher in the elderly (up to $81.2 \%){ }^{9}$
With this context in mind, Tan et al. evaluate the effect of phased, multidisciplinary interventions to reduce PPI overutilisation at a tertiary hospital in Singapore, published in this issue of the Annals. ${ }^{10}$ Sustained behaviour change is challenging. The team employed a framework by Anderson et al. ${ }^{11}$ to target prescriber beliefs, knowledge and attitudes, as well as work environment and cultural factors, to "nudge" physicians towards intended behaviours. The first intervention was the introduction of a PPI deprescribing guide to 4 large prescribing departments within the hospital. The second intervention, introduced 10 months after the first, was the conducting of education sessions for internists at the hospital to highlight the potential harms of inappropriate PPI use.

The retrospective observational study utilised an interrupted time-series analysis to measure doses of oral PPI prescribed per 1,000 prescriptions every month, from 2013 to 2019, as a primary outcome. For safety analysis, the authors explored incident peptic ulcer disease per 1,000 patient-days for the hospital from 2015 to 2018 . These calculations utilised routinely collected data as part of patient care within their hospital database. In addition, a case-notes review was undertaken for patients who had oral PPI deprescribed over a 5-month period (June to October 2017) to explore if restarting or escalation of PPI doses were needed within 6 months of discharge $(\mathrm{N}=262)$.

Interestingly, the study found that PPI utilisation rates were already declining before the first intervention (-77 daily doses/1,000 prescriptions a month, 95\% confidence interval [CI] -105 to -52$)$. This trend accelerated after the first intervention (-303 daily doses/1,000 prescriptions a month, 95\% CI -474 to -131), but reversed after the second intervention $(+405$ daily doses/1,000 prescriptions a month, 95\% CI 231 to 579). On deeper exploration, the rate of inpatient reduction in PPI prescription was steeper than outpatient prescription rates after the first intervention. However, after the second intervention, the rate of inpatient PPI prescriptions started to increase, and the rate

\footnotetext{
${ }^{1}$ National University Hospital, Singapore

${ }^{2}$ Yong Loo Lin School of Medicine, National University Singapore

Correspondence: Division Advanced Internal Medicine, Tower Block 10 $0^{\text {th }}$ Floor, National University Hospital System, $1 \mathrm{E}$ Kent Ridge Rd, Singapore 119228. Email: John_Soong@nuhs.edu.sg
} 
of reduction in outpatient PPI prescription slowed. Overall, the study estimated a reduction of 1,930 daily doses $/ 1,000$ prescriptions a month, and a cost avoidance of SGD87,082 per year.

From the safety analysis, the rate of incident peptic ulcer disease did not change over time, and the casenotes review found that $62.6 \%$ of the patients had remained deprescribed of PPIs at 6 months after discharge. Of the $8.7 \%$ who died, none had a cause attributable to gastrointestinal bleeding. At least $10 \%$ of patients who had PPI treatment restarted had no clear indication documented.

Healthcare systems are dynamic and complex. Interventions rarely happen in isolation. Evaluation of interventions within real-life settings are necessary and meaningful. However, the inability to have rigorous control for environmental and patient factors (e.g. within a randomised clinical trial) often limits both the generalisability of outcomes, and causal inference. The interventions documented in the study occurred after a national campaign for deprescribing, which could explain the already declining rate of PPI prescriptions.

This study highlights the difficulties of enacting sustained behavioural change, and the challenges of measuring improvement and outcomes in the real-world clinical setting. The study utilises routinely collected data as part of patient care, to measure the primary outcome of PPI utilisation rates. This may be interpreted as a surrogate measure for the inappropriate prescribing of PPI. A "floor" effect is to be expected with this surrogate measure. Perfect performance would still produce a PPI prescription rate, though the latter would be clinically indicated. What this "target" should be is not clear, without adjustment for case-mix and clinical indication.

The methodology allows large amounts of data to be interrogated over time. However, as said data were not collected for the expressed purpose of answering this research question, it is subject to several limitations: information bias, not being comprehensive in terms of variables, inaccuracy of diagnostic and prescription coding, its retrospective nature, and having missing data elements not by chance. The study aimed to ameliorate these limitations by complementing the large data approach with a smaller case-notes review. While this study is limited to the acute hospital setting, it is expected that a large proportion of patients may have inappropriate PPIs prescribed in primary care and the community setting.

For a drug, efficacy is not clinical effectiveness. Realworld evaluations can provide meaningful answers to important questions about burden of disease, treatment adherence, cost-effectiveness, and health resource utilisation. Though with limitations, the study adds to our growing understanding of how we can better care for our patients.

\section{REFERENCES}

1. Strand DS, Kim D, Peura DA. 25 Years of Proton Pump Inhibitors: A Comprehensive Review. Gut Liver 2017;11:27-37.

2. Poynard T, Lemaire M, Agostini H. Meta-analysis of randomized clinical trials comparing lansoprazole with ranitidine or famotidine in the treatment of acute duodenal ulcer. Eur J Gastroenterol Hepatol 1995;7:661-5.

3. Prichard PJ, Jones DB, Yeomans ND, et al. The effectiveness of ranitidine in reducing gastric acid-secretion decreases with continued therapy. Br J Clin Pharmacol 1986;22:663-8.

4. Abbas MK, Zaidi ARZ, Robert CA, et al. The Safety of Longterm Daily Usage of a Proton Pump Inhibitor: A Literature Review. Cureus 2019;11:e5563.

5. Aseeri M, Schroeder T, Kramer J, et al. Gastric acid suppression by proton pump inhibitors as a risk factor for clostridium difficileassociated diarrhea in hospitalized patients. Am J Gastroenterol 2008;103:2308-13.

6. Kuipers EJ, Lundell L, Klinkenberg-Knol EC, et al. Atrophic gastritis and Helicobacter pylori infection in patients with reflux esophagitis treated with omeprazole or fundoplication. N Engl J Med 1996; 334:1018-22.

7. Cheung KS, Chan EW, Wong AYS, et al. Long-term proton pump inhibitors and risk of gastric cancer development after treatment for Helicobacter pylori: a population-based study. Gut 2018; 67:28-35.

8. Chia CTW, Lim WP, Vu CKF. Inappropriate use of proton pump inhibitors in a local setting. Singapore Med J 2014;55:363-6.

9. Akram F, Huang Y, Lim V, et al. Proton pump inhibitors: Are we still prescribing them without valid indications? Australas Med J 2014;7:465-70.

10. Tan CJY, Lee SX, Ng TM. The impact of deprescribing interventions on oral proton pump inhibitor utilisation in a Singapore tertiary hospital: A quality improvement initiative. Ann Acad Med Singap 2022;1:8-15.

11. Anderson K, Stowasser D, Freeman C, et aI. Prescriber barriers and enablers to minimising potentially inappropriate medications in adults: a systematic review and thematic synthesis. BMJ Open 2014;4:e006544. 